\title{
User-Centred Design Method for Digital Catalogue Interfaces
}

\author{
Morie Maho Wielfrid ${ }^{1[0000-0003-0426-3046]}$, Marfisi-Schottman Iza ${ }^{2}$, Goore Bi Tra ${ }^{1}$ \\ ${ }^{1}$ Institut National Polytechnique Felix Houphouët-Boigny, 1093 Yamoussoukro, Côte d'Ivoire, \\ ${ }^{2}$ Le Mans Université, EA 4023, LIUM, 72085 Le Mans, France \\ \{maho.morie, bitra.goore\} @inphb.ci \\ iza.marfisieuniv-lemans.fr
}

\begin{abstract}
Digital catalogues must be intuitive and easy to use. However, designing their interfaces is a complex task because there is so much available information and such little space. The choice of search filters, their format, their position, including the way to represent the results, are not trivial decisions. This paper presents the User-Driven Interface Design (UDID) method that offers five steps with specific material to help end users produce mock-up interfaces for digital catalogues. This method recommends letting participants compose their interfaces according to their needs. In this article, we present how the UDID method offered several befits for designing the interface of a Learning Game catalogue. 17 participants followed this method to produce five mock-up interfaces that we then analysed and compared to create the final interface.
\end{abstract}

Keywords: Interface design, User-Centered Design, Digital Catalogue, UX design method, Learning Games.

\section{Introduction}

Digital catalogues are useful to filter a large number of resources in order to find those that meet ones needs. Such catalogues are used in many domains such as education (e.g. Le Catalogue Collectif de France ${ }^{1}$ ) or commerce (e.g. Amazon). Users should be able to find resources without assistance or significant intellectual effort [1]. Therefore, digital catalogues must provide simple and intuitive interfaces [2]. Yet, digital catalogue interface design is a complex task. Available resources in these catalogues are described with metadata that usually contain a lot of information. For example the Learning Object Metadata (LOM) has 69 fields of information such as title, type of resource, age of the public, cost, etc. [3]. However, it would not be relevant to offer a filter for each of these fields, as this would overload the interface. The problem therefore concerns the choice of the few relevant filters and the format that will facilitate the search. The way the resources, resulting from the search, are displayed is also important to help users make a quick choice.

\footnotetext{
${ }^{1}$ https://bbf.enssib.fr/consulter/bbf-2011-02-0071-001
} 
These difficulties appear more marked in domains where there are no satisfying catalogues to draw inspiration from. This is the case for Learning Games (LGs): the existing LG catalogues do not offer filtering systems that facilitate the selection of LGs adapted to teachers' specific needs [4]. To illustrate, the SeriousGamesClassification $^{2}$ catalogue, offers a filter system based on three criteria: the purpose of the LG (e.g. education, information or marketing), the market (e.g. health, communication or politics) and the public (e.g. children, general public or professionals) [5]. The Mobygames $^{3}$ catalogue offers filters based on the platform (e.g. PC, Nintendo), the year of the LG, the game theme (visual, board game or shooter) and the game rating (e.g. ESBR, PEGI). First of all, these catalogues do not comply with the basic UX design models [6]. The filters on SeriousGamesClassification for example, are all in checkbox format, whether the possible values are numerical, textual or require choosing a range of values. The filters of Mobygames are available as links that open up another page, which complicates the search process, because the users are most likely to go back to the first page to try other filters. In addition, the thumbnails that represent the result LGs only contain an image, the title of the game and the year of publication, which is not sufficient to help teachers. Most importantly, the existing catalogues that inventory LGs (e.g. SeriousGamesClassification, Mobygames, Serious Games $F R^{4}$, MIT Step ${ }^{5}$, LearningGamesForKids ${ }^{6}$ ) do not allow to search for LGs depending on their educational goal. This is probably because these catalogues are not primarily intended for teachers. They are designed by video game experts and offer more non-education games than LGs. The existing catalogues therefore do not answer the teacher's need for an intuitive tool to find LGs for their classes.

In a previous study, we show that it is possible to browse LG web sites and automatically collect 23 items of information, compliant with the Learning Games Metadata Definition (LGMD) [7]. These items include the educational topic for which the LG may be relevant, its game type and the platform requirement to play it. Each of these 23 items can be transformed into a filter but there is only room for a few on the catalogue's homepage. Moreover, each filter can be implemented in various formats such as checkbox, drop-down list, radio button etc. Search results (i.e. LGs that meet the search criteria) can also be represented in different ways. In addition, the layout of these filters and results is important to create a good user experience. Given the many choices that need to be made, it seems paramount to consult the end users at the very beginning of the interface design process. Thus, we propose a digital catalogue interface design method, inspired by the user-centred approach [8].

Section II presents a state of the art of interface design methods. In section III and IV we present the UDID method and how it was used for designing a LG catalogue interface. Finally, in section V, we discuss the difficulties observed by teachers when using UDID and the relevance of the proposed interface mock-ups, followed by the conclusion and perspectives.

\footnotetext{
${ }^{2} \mathrm{http}: / /$ serious.gameclassification.com/

${ }^{3} \mathrm{https} / / / \mathrm{www}$.mobygames.com/

${ }^{4}$ https://www.serious-game.fr/

${ }^{5} \mathrm{https}$ ://education.mit.edu/

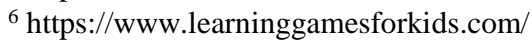




\section{$2 \quad$ Interface Design Methods}

There are several methods for interface design [9]. The UX (User eXperience) design methods, for example, give guidelines on the colours to adopt, the type of widgets and their layout on the interface, etc.[2]. This method is interesting because it allows structuring the web pages content to be more pleasant for the users by highlighting the important content with appropriate style choices. However, these methods do not involve the end users for the beginning [10] and may lead to redesigning the interfaces when the final product is confronted to them, which is very expensive.

The Agile method is an approach that consists in developing applications incrementally by asking the user's appreciation at each design step [11]. This validation is important for further application design because it allows to improve the interface and functionalities but it provides limited information on the interface that users actually want [12]. Indeed, end users will tend to make minimal changes on the already designed interfaces, without thinking of the interface they would really like. When it comes to creating a catalogue interface, one of the most complex tasks lies in the interface organization. Out of the dozens possible ways of organizing the interface, the designers will make one choice that will not necessarily be the end users' choice.

The User-Centred Design (UCD) approach therefore seems to be most suitable because it delegate the interface design and all non-technical parts of application to the end users [13]. It is an interactive approach that allows knowing users' needs and preferences. However, in the case of LG catalogue design, UCD should be implemented with caution because there are no existing satisfactory examples to build on [14]. To solve this problem, we propose a design method adapted from UCD with clearly defined steps and material to help the end users design their ideal interface.

\section{UDID Catalogue Interface Design Method}

The User-Driven Interface Design (UDID) method is inspired by Learning Analytics Dashboard (LAD) method [8], which generates dashboards for learning in which the end users are involved. The UDID method also requires the involvement of volunteer participants who are representative of the catalogues' end users. These participants are mobilized to design the mock-up interfaces themselves and not to evaluate proposed mock-ups. Participants must be organized in teams of three or four to boost creativity but also to encourage them to explain their choices in order to clearly understand their approach. They also need to be guided through this process with a step by step method and specific material.

\subsection{Material for the UDID Method}

The material for the UDID methods consists of:

- A white A3 cardboard that represents a computer screen. One could also choose a phone or tablet depending on the context of use. 
- Filter cards, representing all available product information. For each filter, six formats are available: drop-down list, checkbox, radio button, textbox, word cloud and range sliders (figure 3).

- Post-it notes, which are used to represent thumbnails of the products matching the search. Participants can list the information they want to have for products' summary descriptions.

The size ratio of these items should represent the screen without having to scroll down. The filter cards should show both filter name and format design with possible examples of available information. Each filter should have a card in each available format, unless this format is not suitable for the type of information carried by this filter (figure 3). Finally, participants should have empty post-it notes, pens and markers to modify the given material or create new ones.

\subsection{Five steps of the UDID Method}

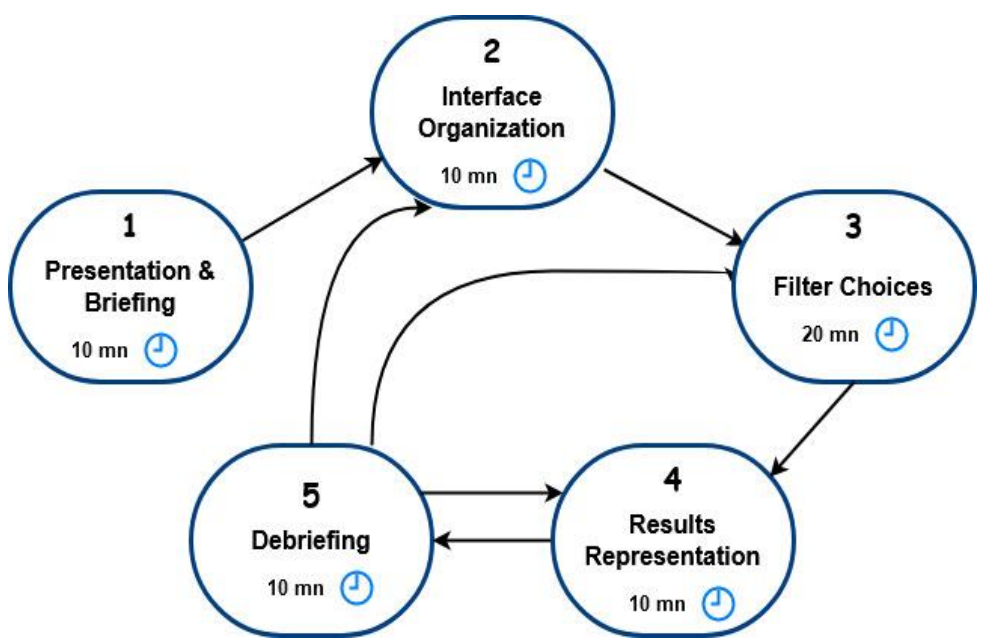

Fig. 1. Five steps of User-Driven Interface Design (UDID) method

The participants follow five steps in which they use the above material (Figure 1):

- Step 1 - The person organizing the design session presents the goals (i.e. create a catalogue interface mock-up) to the participants and explains how to use the material while insisting on the possibility to create new material. It is also important to indicate the time required for each step of the process.

- Step 2 - The participants organize the interface by deciding where to place the search and result areas.

- Step 3 - The participants choose the filter cards they want to place in the search area and determine their layout. To help them, all filters should be visible on the table, grouped by filters types. This step can be quite time-consuming because of the large number of filters. 
- Step 4 - The participants have to determine how they would like to see the research results displayed on the interface. They can use Post-it notes to represent the result thumbnails, on which they can draw the information they would like to see (i.e. description information, image, key words, etc.).

- Step 5 - A debriefing should be carried out at the end of session to help the participants review their choices and make changes to the proposed interface based on new ideas that emerge from the discussions.

It is important to film the design session to help identify the components and displays on which there was immediate consensus and those for which the decision was much more difficult. This information is precious for designing the final mock-up interface. In the next section, we will present how UDID was applied, through several design sessions with teachers, to design a LG catalogue.

\section{Design of a Learning Game Catalogue Interface}

\subsection{Participant Profiles}

The purpose of this catalogue is to allow education stakeholders find LGs adapted to specific teaching needs. Hence, we solicited 17 volunteers with the right profile: 12 teachers, 3 librarians and 2 educational designers. Educational designers are experts in computer technologies for education. Their ability to design and manage training devices is an asset to evaluate a learning resource platform. In addition, they have a good appreciation of how to describe the needs of teachers, since they train them. The librarians chosen were familiar with classic learning resource catalogues, such as those found in libraries and online learning resource platforms. They also recently created the Nantilus ${ }^{7}$ online catalogue for medical learning resources. Although these participants are not representative of the end users, we assumed they would provide us with interesting ideas. The teachers were chosen to represent a diversity of domains and levels: 4 teachers for pre-school and primary school, 4 for middle school and 4 for higher school. None of them was familiar with searching online learning resources. Table 1 summarizes the number of participants for each of the five teams.

Table 1. Distribution of participants by co-design team

\begin{tabular}{lccccc}
\hline & Team 1 & Team 2 & Team 3 & Team 4 & Team 5 \\
\hline Date & 24/01/2020 & $31 / 01 / 2020$ & $07 / 02 / 2020$ & 07/02/2020 & 07/02/2020 \\
\hline Place & Université du Mans & Université de Nantes & CBCG Daloa & CBCG Daloa & CBCG Daloa \\
\hline Nb participants & 2 & 4 & 3 & 4 & 4 \\
\hline $\begin{array}{l}\text { Participant } \\
\text { profiles }\end{array}$ & 1 teacher (high school) & 3 librarians & $\begin{array}{c}\text { 3 teachers } \\
\text { 1 educational designer }\end{array}$ & $\begin{array}{c}\text { 1 educational designer } \\
\text { (high school) }\end{array}$ & $\begin{array}{c}\text { (college) } \\
\text { (preschool) }\end{array}$ \\
\hline
\end{tabular}

We planned three co-design sessions: two in France with team 1 and team 2 and another session in Côte d'Ivoire, with the three remaining teams (Figure 2). Even though

\footnotetext{
${ }^{7}$ https://nantilus.univ-nantes.fr/vufind/
} 
two different researchers carried out the experiments in France and Cote d'Ivoire, we provided the same material and followed the same strict experimental protocol.

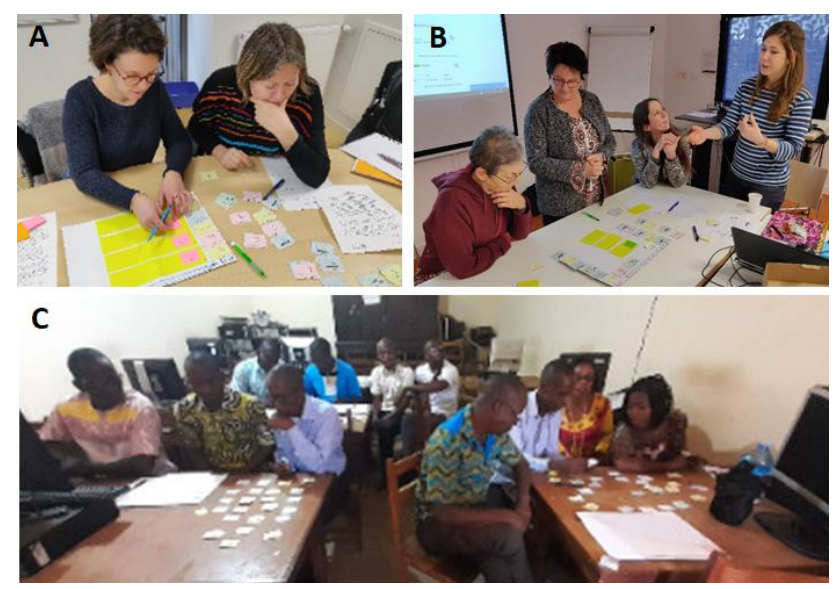

Fig. 2. Co-design sessions in Le Mans (A), Nantes (B), and Daloa (C)

\subsection{Experimentation Material and Protocol}

The filter card we create with the 23 information items of the LGMD metadata model [8]. For each filter, we created up to six card that represented the available formats depending on the type of values (Figure 3 ).

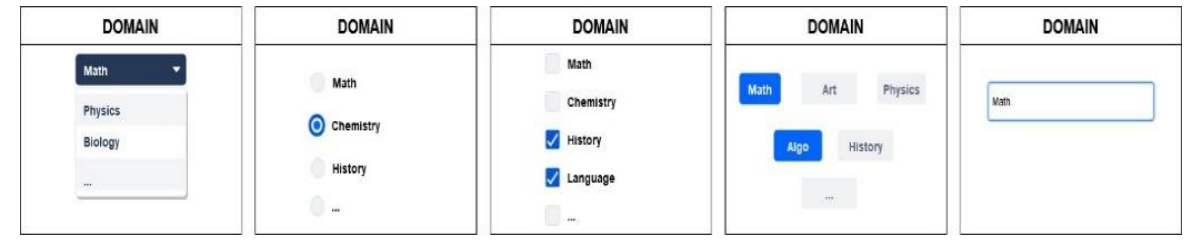

Fig. 3. Different formats for the educational domain filter

The co-design sessions followed the one-hour UDID process. However, all the sessions exceeded this time limit, with some teams working up to two hours. The debriefing was particularly rich and provided valuable insight on the co-design method. We filmed all the sessions in order to analyse each step and points of conflict. This was crucial to design the final catalogue interface, based on the five mock-up interfaces designed by the teams (Figure 4). In the next section, we analyse these mock-up interfaces and explain how they helped us answer the four questions for designing digital catalogues: which filters should be chosen, in what format should they be, how should the interface be organized and how should the results be presented?

\subsection{Results of Interface Design Sessions}

In this section, we analyse the mock-up interfaces designed by the five teams and decide which design choices should be kept for the final LG catalogue interface. 


\section{Interface Organization}

- We observed almost the same result for all teams regarding the web page organization: four teams placed the search area on the left, in which they placed filters vertically. For the result area, four out of five teams create thumbnails in a row (Figure 4). We therefore decided to choose the interface organization that most of the teams used. In addition, we add a sorting area, above the result area, as proposed by Team 2 , composed of librarians, who already had experience in designing such catalogues.
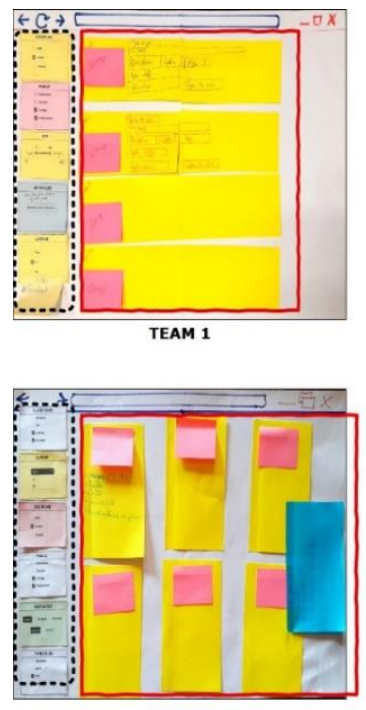

TEAM 3
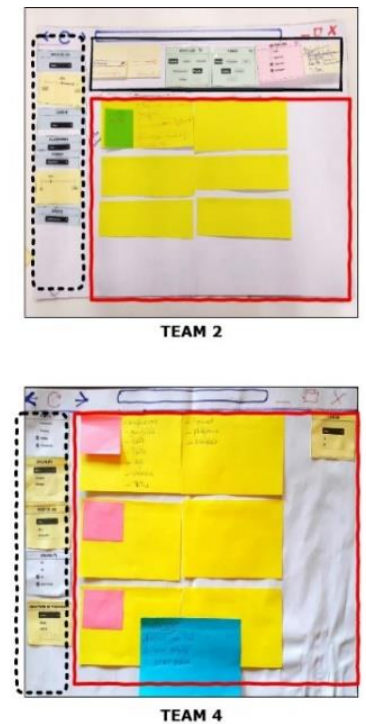
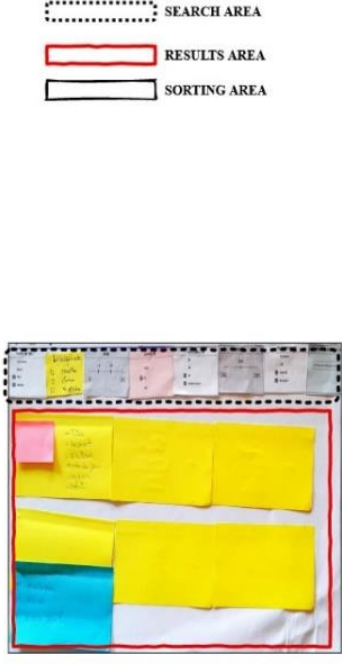

TEAM 5

Fig. 4. Teams' Mock-ups in Co-design sessions

\section{Choice of Search Filters}

. The analysis of the five mock-up interfaces shows that each team chose an average of six filters for the search area. Team 2 also proposes four additional filters, in a new area labelled "sorting area". For the final mock-up, we decided to keep the filters Language, Platform requirements, Public and Domain that were chosen by at least three teams (in yellow in table 2). We also added the filters Keywords and Age, considered as sub-criteria of the filters Domain and Public by the participants. In addition, we added the filters Game Mode and Cost, chosen by two teams, but with default options already activated. We also chose to place the filter Date in sorting area. Finally, we added the Motivation filter, which carries essential information, especially in primary and pre-school level, as expressed by team 5, composed of this profile.

Table 2. Filters chosen per team

\begin{tabular}{lcccccc}
\hline Filters & Team 1 & Team 2 & Team 3 & Team 4 & Team 5 & Total \\
\hline Title & & & & & & 0 \\
\hline Language & $\mathrm{X}$ & $\mathrm{X}$ & $\mathrm{X}$ & $\mathrm{X}$ & $\mathrm{X}$ & 5 \\
\hline Description & & & & & & 0 \\
\hline Keywords & $\mathrm{X}$ & $\mathrm{X}$ & & & & 2 \\
\hline
\end{tabular}




\begin{tabular}{|c|c|c|c|c|c|c|}
\hline Version & & & & & & 0 \\
\hline Contribution & & & & & & 0 \\
\hline Date & & $x$ & & $x$ & & 2 \\
\hline Format & & $x$ & & & & 1 \\
\hline Size & & & & & & 0 \\
\hline Location & & & & & & 0 \\
\hline Platform requirements & & $x$ & & $x$ & $x$ & 3 \\
\hline Public & $\mathrm{x}$ & $x$ & $\mathrm{x}$ & $\mathrm{x}$ & $x$ & 5 \\
\hline Age & $\mathrm{x}$ & & & $x$ & & 2 \\
\hline Motivation & & $x$ & & & $x$ & 2 \\
\hline Knowledge Validation & & & & $\mathrm{x}$ & & 1 \\
\hline Game Type & & & & $x$ & $x$ & 2 \\
\hline Game Mode & & $x$ & $x$ & & & 2 \\
\hline Domain & $x$ & & $x$ & $\mathrm{x}$ & $x$ & 4 \\
\hline Progress Indicators & & & $\mathrm{x}$ & & & 1 \\
\hline Cost & $x$ & $x$ & & & & 2 \\
\hline Rights & & $x$ & & & & 1 \\
\hline Gameplay & & & $\mathrm{x}$ & $\mathrm{X}$ & & 2 \\
\hline Rating & & & & & & 0 \\
\hline
\end{tabular}

\section{Search Filter Formats}

. The analysis of the mock-up interfaces shows that the teams chose different formats for a given filter. We therefore followed the following tendencies. In all, the drop-down list format was used the most ( 7 times for the filters chosen above). It was particularly appreciated for filters containing over three values and requiring a single choice such as Domain and Public, because it takes up little space on the interface. The checkbox format was also use 7 times. This format was uses for the filter Platform, which allows several choices of values. The range sliders format was preferred for filters with numerical information such as Age. The text box format was chosen for filters with free information such as Keywords. Finally, filters containing two values such as Language, Game Mode and Cost were placed in the sorting area in radio button format with default options.

\section{Presentation of LG Research Results}

. Concerning the design of the LG thumbnails on Post-it notes, all teams reserved space for an illustrative image of the LG with several information fields. The information presented was quite diverse with 17 fields chosen out of 23. However, all teams chose Title and three teams chose Gameplay and Cost. The other fields were selected by maximum two teams and the films show that their choice was not particularly motivated. For the final interface, we therefore decided to use Title, Gameplay and Cost and add the fields used in the search area (i.e Domain, Keywords, Public, Age and Platform) since this information can help them selected LGs that meets their search criteria. Finally, we added information on the Motivation and Progress Indicator as participants mostly showed interest about the LG's pedagogical characteristics. We arranged the fields according to space that information could occupy since participants did not give importance to information's order or appearance (Figure 5). 


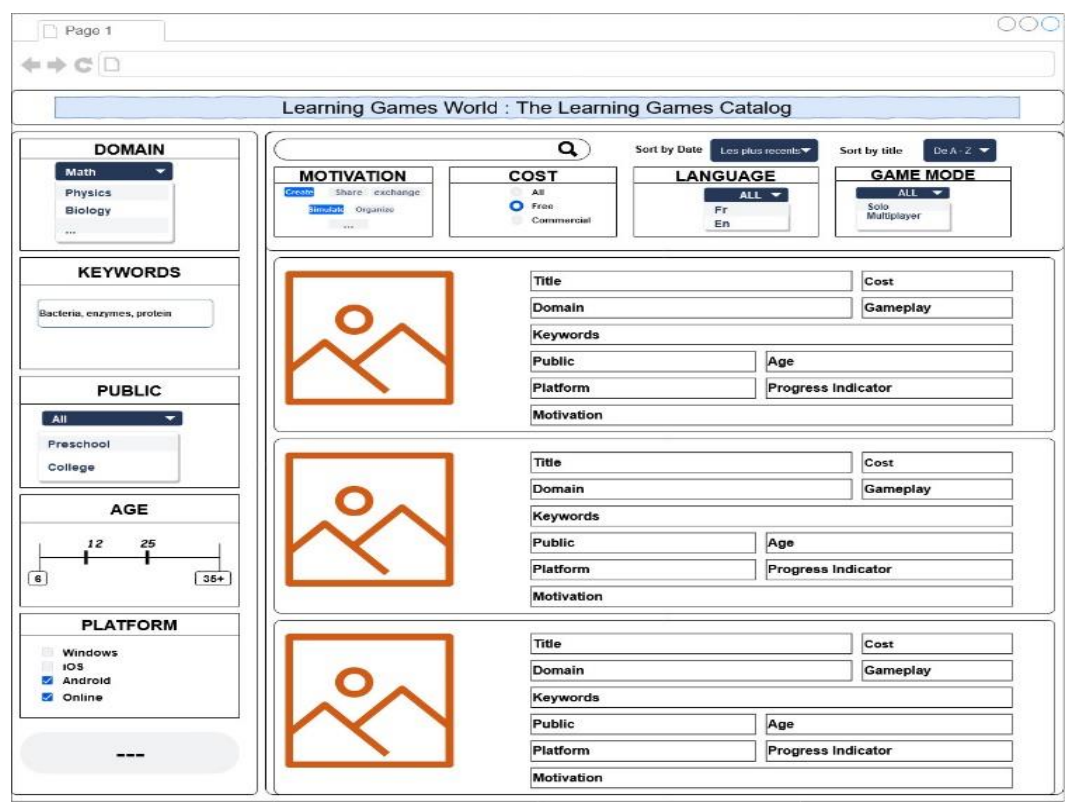

Fig. 5. Final interface mock-up resulting from the UDID method

\subsection{Discussion}

As we noted throughout the discussions with the participants, all the teams managed to apply the UDID method without difficulty and found it was a helpful for designing interfaces. They particularly appreciated the use of paper materials that encouraged them to take part in the design sessions. Being able to customize and move the cards seemed to help them express their needs and organize the interface the way they wanted. The team of librarians also expressed a preference for this approach rather than the Agile method they have recently used to design their educational resource catalogue. However, we identified several limitations. First, the time allocated to each design session was clearly insufficient. Choosing the filters and placing them on the interface was the most time-consuming. The format of the filter were also subject to a lot of discussion, which was not unanimous in all teams. A solution may be to preselect filter formats based on the type of content. Another delicate choice we made was to involve five teams. It might be better to involve fewer teams to simplify the final design choices, based on their mock-ups that were sometimes quite different. This might also be because the participants were grouped by profile. This fact underlines why it is important to film all sessions in order to understand the dynamics behind the participants' choices and their priorities regarding the search filters.

\section{Conclusion and Perspectives}

The User-Driven Interface Design (UDID) method allows us to meet the challenge of designing a Learning Games (LG) catalogue interface. Thanks to its clear step-by-step 
method and the material it provided, five teams of teachers and other educational stakeholders were able to design mockup models of the interface. By comparing the similarities of these mockups and analyzing the discussions that the participants had during the design phase, we were able to design a final interface for the LG catalogue.

Nevertheless, we need to verify that the final interface truly allows teachers to find the relevant LGs quickly. To do this, we will carry out another experimentation with the final LG catalogue. UDID could also be adapted to the design of mobile application interfaces. Indeed, it raises similar challenges such as choosing and organizing a lot of potential information in on a very small screen.

\section{References}

1. Dos Santos, F.A., Souto, V.T.: Graphic design and user-centred design: designing learning tools for primary school. Int. Journal of Tech. and Design Edu. 29, 999-1009 (2019).

2. Kurniawan, S., Zaphiris, P.: Research-derived web design guidelines for older people. In: Proceedings of the SIGACCESS conf. pp. 129-135. ACM (2005).

3. Neven, F., Duval, E.: Reusable Learning Objects: A Survey of LOM-based Repositories. In: Proceedings of the Conf. on Multimedia. pp. 291-294. ACM (2002).

4. Morie, M.W. et al.: Information Extraction Model to Improve Learning Game Metadata Indexing. ISI. 25, 11-19 (2020).

5. Djaouti, D., Alvarez, J., Jessel, J.-P.: Classifying Serious Games: the G/P/S model. Handbook of Research on Improving Learning and Motivation through Educational Games: Multidisciplinary Approaches. (2011).

6. Leavitt, M.O., Shneiderman, B.: Based web design \& usability guidelines. Background and Methodology. (2006).

7. Morie, M.W. et al., B.T.: LGMD: Optimal Lightweight Metadata Model for Indexing Learning Games. In: Smart Applications and Data Analysis. pp. 3-16. Springer (2020).

8. Ines, D., et al:: User Centered Approach for Learning Analytics Dashboard Generation. In: Proceedings of Conf. on Comp. Sup. Edu. pp. 260-267., Heraklion, Greece (2019).

9. De Troyer, O.M.F., Leune, C.J.: WSDM: a user centered design method for Web sites. Computer Networks and ISDN Systems. 30, 85-94 (1998).

10. Sutcliffe, A.: Assessing the reliability of heuristic evaluation for Web site attractiveness and usability. In: Proceedings of the Hawaii Int. Conf. pp. 1838-1847 (2002).

11. Maleki, N.G., Ramsin, R.: Agile Web Development Methodologies: A Survey and Evaluation. In: Software Engineering Research, Management and Applications. pp. 1-25. Springer International Publishing, Cham (2018).

12. Torrecilla-Salinas, et al: : Estimating, planning and managing Agile Web development projects under a value-based perspective. Inf. \& Software Tech. 61, 124-144 (2015).

13. Schnall, R., et al: A user-centered model for designing consumer mobile health (mHealth) applications (apps). Journal of Biomedical Informatics. 60, 243-251 (2016).

14. Vredenburg, K. et al., T.: A survey of user-centered design practice. In: Proceedings of SIGCHI. pp. 471-478. ACM, Minneapolis, Minnesota, USA (2002). 